\title{
Controlling the elongational flow behavior of complex shear-thinning fluids without affecting shear viscosity
}

\author{
Walter Oswald $^{1} \cdot$ Norbert Willenbacher $^{1}$
}

Received: 13 May 2019 / Revised: 28 August 2019 / Accepted: 28 August 2019

(C) Springer-Verlag GmbH Germany, part of Springer Nature 2019

\begin{abstract}
Complex flow fields including high elongational deformation occur in numerous industrial processes such as spraying, coating, fiber spinning, and screen or inkjet printing. Fully exploiting the potential of these technologies suffers from a lack of knowledge regarding how elongational flow properties of the processed fluids affect the results of these operations. Here, we present two strategies that allow for varying the elongational flow behavior independent of shear rheology. First, two acrylic thickener solutions that differ with respect to the fraction of hydrophobic co-monomers and hence with respect to their degree of interand intramolecular hydrophobic association were mixed to vary the elongational relaxation time, as determined using capillary breakup elongational rheometry (CaBER), by almost two orders of magnitude without affecting shear viscosity of these solutions in a wide, processing-relevant shear rate range. Second, a substantial increase in the elongational flow resistance was achieved by adding a small amount of plate-like particles without affecting the shear viscosity of these thickener solutions. A fourfold increase of the elongational relaxation time was observed upon the addition of $3.5 \mathrm{vol} . \%$ of glass flakes to such a highly shear-thinning system. A similar effect was also observed for an industrial waterborne automotive basecoat due to added aluminum flakes. This work may be useful for product development since the control of extensional viscosity can improve technological applications, and the introduced model systems may therefore be used for systematic, goal-oriented investigations of the relevance of elongational flow properties in the technological processes mentioned above.
\end{abstract}

Keywords Elongational flow $\cdot$ CaBER $\cdot$ Acrylic thickener $\cdot$ Hydrophobic associations $\cdot$ Plate-like particles $\cdot$ Glass flakes

\section{Introduction}

In a broad variety of technical processes, fluids are subject to complex flows that often include components of elongational deformation. For Newtonian fluids, extensional viscosity is strain rate-independent irrespective of flow kinematics, but the ratio of extensional to shear viscosity depends on the type of flow (Trouton 1906). However, many industrially relevant materials are viscoelastic, shear-thinning fluids. For these nonNewtonian fluids, extensional viscosity generally depends not

Walter Oswald

Walter.Oswald@kit.edu

Norbert Willenbacher

Norbert.Willenbacher@kit.edu

1 Karlsruhe Institute of Technology, Institute for Mechanical Process Engineering and Mechanics, Gotthard-Franz-Straße 3, Geb. 50.31, 76131 Karlsruhe, Germany only on the strain rate but also on the total strain, and often a steady-state is hardly accessible. Furthermore, extensional viscosity can be orders of magnitude higher than the shear viscosity, and strain hardening can be observed even for shearthinning fluids (Spearot and Metzner 1972; Meissner 1985). Therefore, the adjustment of shear and elongational viscosity independently according to processing and application demands is an important technological challenge. Here, we will demonstrate how to vary elongational relaxation time in a broad range without significantly affecting shear flow properties.

This work is motivated by the extensive literature on the effect of extensional viscosity on atomization. For example, it has been shown that liquid breakup and droplet formation are delayed by an increase in this rheological parameter (Mansour and Chigier 1995; Thompson and Rothstein 2007). Furthermore, an increase in the elongational flow resistance leads to an increase in the droplet diameter (Dexter 1996; Xing et al. 1999; Ochowiak et al. 2012). Fernando et al. (2000) showed that roll misting can be prevented using fluids with 
low extensional viscosity. The tendency of the droplet bounce after impact is decreased by an increase in the extensional viscosity (Bergeron et al. 2000; Williams et al. 2008). Furthermore, elongational flow resistance supports the decrease in fluid retraction (Bergeron et al. 2000; Bartolo et al. 2007). Many studies also suggest that inkjet printing (Lampe et al. 2005; Vadillo et al. 2010; Hoath et al. 2014; Jimenez et al. 2018) and fiber spinning (Hyun 1999; Gupta and Chokshi 2017) are strongly affected by the elongational viscosity of the processed fluids.

Coatings, inks, or adhesives are formulated as low viscosity multiphase fluids. Typically, such formulations exhibit strong shear thinning, often due to the presence of the socalled rheology modifiers or thickeners, i.e., polymers with high molecular weight and/or complex topology and supramolecular aggregation structure. Typically, they also include solid particles with different shapes in order to guarantee the desired application properties.

Several techniques were developed for characterizing elongational flow behavior, such as fiber spinning (Spearot and Metzner 1972; Papanastasiou et al. 1987), entrance flows (Cogswell 1972; Kwag and Vlachopoulos 1991; Padmanabhan et al. 1997), opposed jet (Fuller et al. 1987), or filament stretching (Tirtaatmadja and Sridhar 1993; Spiegelberg et al. 1996; Anna et al. 2001) rheometry. The capillary breakup elongational rheometer (CaBER) (Entov and Hinch 1997; McKinley and Tripathi 2000) is particularly suitable for low viscosity fluids and provides a predominantly uniaxial elongational flow (Rodd et al. 2005; Tropea et al. 2007). In this device, an unstable filament is created by rapidly stretching a fluid drop placed between two circular plates. The subsequent thinning of the created filament is controlled by the balance between inertia, viscoelastic, and capillary forces. Apparent extensional viscosity and elongational relaxation time $\lambda_{\mathrm{e}}$ can be calculated from the thinning dynamics of the filament.

Polymer solutions can be classified according to their thermodynamic properties. Depending on the polymer concentration, the dilute, semidilute, and concentrated regimes are distinguished (Teraoka 2002). Capillary thinning of dilute solutions was investigated in many previous studies $(\mathrm{Ng}$ et al. 1996; Bazilevskii et al. 1997; Entov and Hinch 1997; Stelter et al. 2000, 2002; Gupta et al. 2000; Anna and McKinley 2001; Christanti and Walker 2001, 2002; Rothstein and McKinley 2002; Rodd et al. 2005; Tirtaatmadja et al. 2006; Clasen et al. 2006; Tembely et al. 2012; Keshavarz et al. 2015; Greiciunas et al. 2017). For polystyrene in an oligomeric styrene solution, Anna and McKinley (2001) showed that $\lambda_{\mathrm{e}}$ show molecular weight dependence according to the Zimm model. Stelter et al. (2000) observed the dependence of $\lambda_{\mathrm{e}}$ on polymer concentration. In some studies, even a power-law scaling was found between $\lambda_{\mathrm{e}}$ and the polymer concentration (Tirtaatmadja et al. 2006; Clasen et al. 2006; Bazilevsky et al.
2011). Clasen (2010), Arnolds et al. (2010), and later Sachsenheimer et al. (2014) compared $\lambda_{\mathrm{e}}$ with the terminal shear relaxation time $\lambda_{\mathrm{s}}$ for PS and PEO solutions in the semidilute and concentrated regime, revealing that $\lambda_{\mathrm{e}}<\lambda_{\mathrm{s}}$. Additionally, Sachsenheimer et al. (2014) found an exponential decay for the plot of $\lambda_{\mathrm{e}} / \lambda_{\mathrm{s}}$ vs. c/c* irrespective of the type and molecular weight of the polymer, solvent viscosity, or temperature. Very low ratios $\lambda_{\mathrm{e}} / \lambda_{\mathrm{s}}<0.1$ at $\mathrm{c}>\mathrm{c}^{*}$ were also found for aqueous solutions of acrylic polymeric thickeners known to form large intermolecular aggregates via hydrophobic interactions (Kheirandish et al. 2008).

The flow behavior of a polymer melt including nonBrownian, monodisperse polystyrene spheres was investigated by Le Meins et al. (2003). Both shear and extensional viscosity were found to follow the phenomenological Krieger-Dougherty equation describing the concentration dependence of viscosity, i.e., an increase in the fraction of nonBrownian particles resulted in a similar increase in the stress level in shear and elongational deformation. By contrast, suspensions of anisotropic particles show greater resistance to elongational than to shear deformation. Theoretical work on stress occurring during elongational deformation of dilute and semidilute suspensions of rod-like particles was performed by Batchelor (1971) and Shaqfeh and Fredrickson (1990). Both studies predicted a sharp increase of extensional viscosity with increasing aspect ratio (AR) in good agreement with experimental results (Kizior and Seyer 1974; Mewis and Metzner 1974; Weinberger and Goddard 1974).

In summary, there is a large body of literature dealing with the elongational flow behavior of polymer solutions or suspensions. Nevertheless, it is still unclear how to independently control the elongational and shear flow resistances of low viscosity shear-thinning fluids typically used in numerous printing and coating operations. Therefore, we present two different strategies that allow for the variation of the elongational flow resistance at essentially constant shear flow properties. The first approach is based on the observations of Kheirandish et al. (2009) who demonstrated that increasing the fraction of a crosslinked polymer in a mixture of two otherwise similar alkali swellable emulsion (ASE) type thickeners leads to a strong increase in the shear relaxation time, while the elongational time decreases. Therefore, we varied the amount and mixing ratio of two such commercial acrylic ASE-type thickeners that differ in their density of hydrophobic association (physical crosslinks) in order to vary the elongational relaxation time without strongly affecting shear rheology. The second approach is based on the addition of plate-like particles with high aspect ratio to a shear-thinning thickener solution. Prior work on the suspensions of oblate particles indicated that shear viscosity hardly depends on the particle concentration at low volume fractions (SantamaríaHolek and Mendoza 2010; Mueller et al. 2010). Here, we will demonstrate that in a wide shear rate range, even small 
fractions $\left(\varphi_{\mathrm{f}}<3.5\right.$ vol. $\left.\%\right)$ of plate-like particles can lead to a strong increase in the elongational relaxation time while leaving shear viscosity unchanged.

Elongational flow behavior of the investigated solutions and suspensions was characterized using CaBER experiments supported by particle image velocimetry (PIV) in order to determine the true velocity field within the thinning filament. Shear flow properties were thoroughly determined to employ rotational shear and capillary rheometry and oscillatory squeeze flow, thus covering a broad range of shear rates $\left(10^{-2} \mathrm{~s}^{-1}<\dot{\gamma}<10^{6} \mathrm{~s}^{-1}\right)$ in a steady flow and a broad range of frequencies in small amplitude oscillatory shear $\left(10^{-2} \mathrm{rad} \mathrm{s}^{-1}<\omega<10^{4} \mathrm{rad} \mathrm{s}^{-1}\right)$.

The goal of this paper is to demonstrate how elongational relaxation time can be controlled without affecting the shear flow properties for model fluids consisting only of two or three components but closely resembling the absolute viscosity values and shear-thinning properties, e.g., of waterborne automotive coatings. To confirm the technical relevance of these investigations, we also present results for automotive basecoats including various amounts of aluminum flakes.

\section{Material}

Aqueous solutions of Sterocoll@ HT and Sterocoll@ XT (BASF SE, Ludwigshafen, Germany) were investigated. These commercial thickeners are provided as alkalisoluble dispersions made via emulsion polymerization. The solids content of the provided Sterocoll HT dispersion is $40 \mathrm{wt} \%$, Sterocoll XT was delivered with a solids content of $26 \mathrm{wt} \%$, and both dispersions show a $\mathrm{pH}$ of approximately 3 . Both thickeners are copolymers including ethyl acrylate and carboxylic acids. Both types of thickeners are characterized by intra- and intermolecular aggregation due to the random occurrence of hydrophobic segments along the polymer backbone. This gives rise to their unique flow properties (Kheirandish et al. 2008, 2009). The two polymers differ in the fraction of the hydrophobic monomer, which is higher for Sterocoll HT than for Sterocoll XT. Consequently, the density of intra- and intermolecular hydrophobic associations is higher for Sterocoll HT and these molecules are more stiff and less flexible in solution than the molecules of Sterocoll XT. Stock solutions were prepared by diluting Sterocoll HT and Sterocoll XT dispersions to $2.3 \mathrm{wt} . \%$ and $1.4 \mathrm{wt} . \%$, respectively, with distilled water and $1 \mathrm{M} \mathrm{NaOH}$, which was added in order to adjust the solution to $\mathrm{pH}$ 8. During titration, the weak acrylic or methacrylic acid groups start dissociating, resulting in the formation of highly viscous, transparent polymer solutions. These stock solutions were stirred for $1 \mathrm{~h}$ and diluted to the desired concentrations or mixed with each other as summarized in Table 1.
Table 1 Composition of Sterocoll mixtures

\begin{tabular}{lll}
\hline Designation & $\mathrm{c}($ Sterocoll HT)/wt.\% & $\mathrm{c}($ Sterocoll XT)/wt.\% \\
\hline S1 & 2.3 & 0 \\
S2 & 2.0 & 0.15 \\
S3 & 1.8 & 0.22 \\
S4 & 1.7 & 0.3 \\
S5 & 1.6 & 0.4 \\
\hline
\end{tabular}

Glass flakes of Luxan CFX C001 (Eckart GmbH, Hartenstein, Germany) were dispersed into the mixed Sterocoll solution $\mathrm{S} 3$ at different volume fractions $\varphi_{\mathrm{f}}=0.6$, $1.3,2.0$, and 3.5 vol.\%. Moreover, we have dispersed aluminum flakes Alu-Stapa Hydrolux 600 (Eckart GmbH, Hartenstein, Germany) into an industrial waterborne basecoat provided by BASF Coatings GmbH (Münster, Germany) at volume fractions $\varphi_{\mathrm{f}}=1.0,2.0$, and $3.0 \mathrm{vol} . \%$. The geometric dimensions of the particles are shown in Table 2.

\section{Experimental methods}

\section{Rheological characterization}

Rotational rheometry measurements were performed using a HAAKE MARS II instrument (Thermo Fischer Scientific, Karlsruhe, Germany) equipped with a DIN-Z20 coaxial cylinder system. Steady shear flow curves were determined by performing controlled shear stress tests $(\tau=0.2-200 \mathrm{~Pa})$ for Sterocoll mixtures and controlled shear rate tests $(\dot{\gamma}=0.1-$ $1000 \mathrm{~s}^{-1}$ ) for the automotive waterborne basecoat. Small amplitude oscillatory shear (SAOS) experiments were performed at a constant shear stress amplitude of $\tau_{0}=0.1 \mathrm{~Pa}$ in the frequency range from $\omega=0.01$ to $10 \mathrm{rad} \mathrm{s}^{-1}$. For all of the measurements, the temperature was adjusted to $\mathrm{T}=20^{\circ} \mathrm{C}$.

A home-built piston-driven capillary rheometer equipped with a circular die (radius $r_{\text {cap }}=0.25 \mathrm{~mm}$, length $l_{\text {cap }}=$ $40 \mathrm{~mm}$ ) was used to cover the shear rate range of $3 \cdot 10^{2} \mathrm{~s}^{-1}$ $<\dot{\gamma}<3 \cdot 10^{5} \mathrm{~s}^{-1}$. The shear rate is calculated from the preselected volumetric flow rate $\dot{V}$ and shear stress is obtained from the resulting pressure drop $\Delta \mathrm{p}$ recorded with a M30-6-HB01C-4-4-02130X000X00 transducer (Gefran S.p.a., Provaglio d'Iseo, Italy). These experiments were performed at $25^{\circ} \mathrm{C}$.

Table 2 Geometric dimensions of particles

\begin{tabular}{lllll}
\hline Type of particle & $\begin{array}{l}d_{10} / \\
\mu \mathrm{m}\end{array}$ & $\begin{array}{l}d_{50} / \\
\mu \mathrm{m}\end{array}$ & $\begin{array}{l}d_{90} / \\
\mu \mathrm{m}\end{array}$ & $\mathrm{AR}$ \\
\hline Glass flakes & $10-20$ & $25-35$ & $55-65$ & 25 \\
Aluminum flakes & 5 & 15 & 33 & 90 \\
\hline
\end{tabular}


The storage and loss modulus data at high frequencies between $10^{1} \mathrm{rad} \mathrm{s}^{-1}<\omega<10^{4} \mathrm{rad} \mathrm{s}^{-1}$ were obtained using oscillatory squeeze flow rheometry employing a so-called Piezoelectric Axial Vibrator (PAV) (Crassous et al. 2005). The sample is placed in the gap with a height $h_{\mathrm{PAV}}=31 \mu \mathrm{m}$ between two steel plates $20 \mathrm{~mm}$ in diameter. A dynamic squeeze flow is excited by four piezoelectric transducers that are attached to the copper tube glued to the lower plate. The response signal is received by four other piezo elements. Finally, $\mathrm{G}^{\prime}$ and $\mathrm{G}^{\prime \prime}$ are calculated from the corresponding signal amplitude and phase shift data.

Elongational flow behavior was characterized using a Haake CaBER 1 instrument (Thermo Fischer Scientific, Karlsruhe, Germany). Here, the sample is placed between two parallel plates with a diameter $D=6 \mathrm{~mm}$. The upper plate was displaced from the initial height of the gap $h_{i}=2 \mathrm{~mm}$ to the final height $h_{f}=10 \mathrm{~mm}$ within a strike time of $t_{s}=40 \mathrm{~ms}$. The strain rate $\dot{\varepsilon}$ was determined from the time-dependent decrease of the diameter $D(t)$ as follows (McKinley and Tripathi 2000):

$\dot{\varepsilon}=-\frac{2}{D(t)} \frac{d D(t)}{d t}$

Elongational deformation at the midpoint of the filament can be considered as purely uniaxial. Using the FENE dumbbell model for dilute polymer solutions, Entov and Hinch (1997) analyzed the nonlinear dynamics and determined an exponential decay of the filament diameter in the elastocapillary regime. In this type of capillary thinning, elongational relaxation time $\lambda_{\mathrm{e}}$ is related to the constant strain rate according to:

$\lambda_{\mathrm{e}}=\frac{2}{3 \dot{\varepsilon}}$

The CaBER device used here is equipped with a highspeed camera Fastcam 1024 PCI (Photron, Tokyo, Japan), a telecentric objective and a telecentric backlight illumination (Niedzwiedz et al. 2009). The images were recorded with a frame rate of $1000 \mathrm{fps}$ at a resolution of $1024 \times 1024$ pixels. This enabled us to analyze the shape of the necking thread and to accurately monitor diameter decay during the filament thinning. These experiments were performed at ambient temperature.

\section{Particle image velocimetry}

To visualize the true velocity field during capillary thinning, PIV measurements were performed in a horizontally stretched filament (Recktenwald et al. 2019). Therefore, we added 0.01 wt. \% of fluorescent green polystyrene particles (Banglabs Inc., Fishers, USA) with a diameter of $0.96 \mu \mathrm{m}$ to the acrylic thickener solutions including glass flakes with the excitation and emission wavelength of $480 \mathrm{~nm}$ and $520 \mathrm{~nm}$, respectively. The filament stretching device is similar to the tilted CaBER (Sachsenheimer et al. 2012). The sample was placed between two plates with $\mathrm{D}=4 \mathrm{~mm}$ and stretched horizontally from $h_{i}=1 \mathrm{~mm}$ to $h_{f}=4 \mathrm{~mm}$ within $t_{s}=500 \mathrm{~ms}$. Particle flow was observed using an inverted fluorescence microscope AxioObserver D (Carl Zeiss AG, Jena, Germany) equipped with a sCMOS Zyla X camera (Andor Technology Ltd., Belfast, Northern Ireland, $2048 \times 2048$ pixels, up to $50 \mathrm{fps}$ ) and a long working distance lens Nikon LU-Plan (Nikon Corporation, Tokyo, Japan, 20X, NA = 0.4). The particles were illuminated using a constant LED light source Colibri (Carl Zeiss AG, Jena, Germany, $470 \mathrm{~nm}$ ). Since it takes several seconds to stretch the filament and determine the correct focus, it was not possible to perform the measurements for the samples described above. Instead, we prepared another Sterocoll suspension S6 with 1.0 wt.\% Sterocoll HT and $1.0 \mathrm{wt}$ \% Sterocoll XT including glass flakes with $\varphi_{\mathrm{f}}=0.6 \mathrm{vol} . \%$ and $3.5 \mathrm{vol} . \%$, respectively. The lifetime of these samples during capillary thinning was approximately $30 \mathrm{~s}$ and the flow field was analyzed with an open-source PIV software (Thielicke and Stamhuis 2014).

\section{Results and discussion}

\section{Approach 1: mixture of acrylic thickeners differing in their degree of hydrophobic association}

This approach is based on the fact that two acrylic thickeners differ in the fraction of copolymerized ethyl acrylate and carboxylic acids and hence in their degree of hydrophobic association. Sterocoll HT exhibits more intra- and intermolecular associations, thus forming a highly elastic gel with an extended shear relaxation time, but the hardly deformable molecules show little resistance to elongational deformation. By contrast, Sterocoll XT includes a lower fraction of the hydrophobic monomer and hence less internal associations. These molecules are more flexible and provide extended lifetimes during uniaxial filament thinning.

The shear viscosity of these thickeners at various concentrations in neutralized aqueous solutions as a function of shear rate is shown in Fig. 1. The flow curves of the pure Sterocoll HT solutions closely resemble the characteristic degree of shear thinning and the high shear viscosity level typically observed in automotive waterborne basecoats. Similar to the commercial basecoats, the flow curves of the Sterocoll HT solutions do not approach a limiting zero shear viscosity in the investigated shear rate range and apparent yield stress $\tau_{\mathrm{y}} \approx$ $2 \mathrm{~Pa}$ can be estimated that increases weakly with Sterocoll HT concentration. The pronounced shear-thinning behavior is attributed to the breakup of the hydrophobic aggregates due to the applied shear stress. A decrease in the polymer 
Fig. 1 Shear viscosity $\eta$ as a function of shear rate $\dot{\gamma}$ for aqueous thickener solutions with different concentration of a Sterocoll HT and b Sterocoll XT. Insert in a yield stress $\tau_{\mathrm{y}} \mathrm{vs}$. concentration of Sterocoll HT (SHT) and b zero shear viscosity $\eta_{0}$ vs. concentration of Sterocoll XT (SXT)

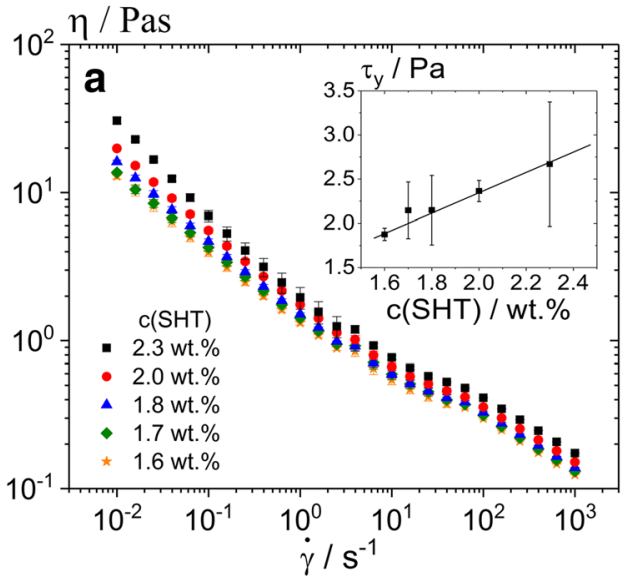

concentration leads to a weak decrease in shear viscosity that is slightly more pronounced at low shear rates than at high shear rates. By contrast, the flow curves of the investigated Sterocoll XT solutions approach zero shear viscosity at shear rates $\dot{\gamma}<1 \mathrm{~s}^{-1}$ and shear thinning is less pronounced than for Sterocoll HT, because Sterocoll XT exhibits fewer hydrophobic intermolecular associations, i.e., the effective molecular weight is lower than for Sterocoll HT. The zero shear viscosity scales linearly with thickener concentration indicating that these solutions are in the dilute regime (Rubinstein and Colby 2003).

Next, we have studied the Sterocoll mixtures listed in Table 1. We mixed both types of thickeners such that Sterocoll HT in the ascending order of concentration was combined with Sterocoll XT in the descending order. As demonstrated in Fig. 2, all of the mixtures show similar shear viscosity for a broad range of shear rates. In particular, for the shear rates $\dot{\gamma}>10^{3} \mathrm{~s}^{-1}$, the absolute values are almost independent of sample composition. In this shear rate range, solution viscosity is mostly determined by stress relaxation of the short-chain segments and hence, depends mainly on

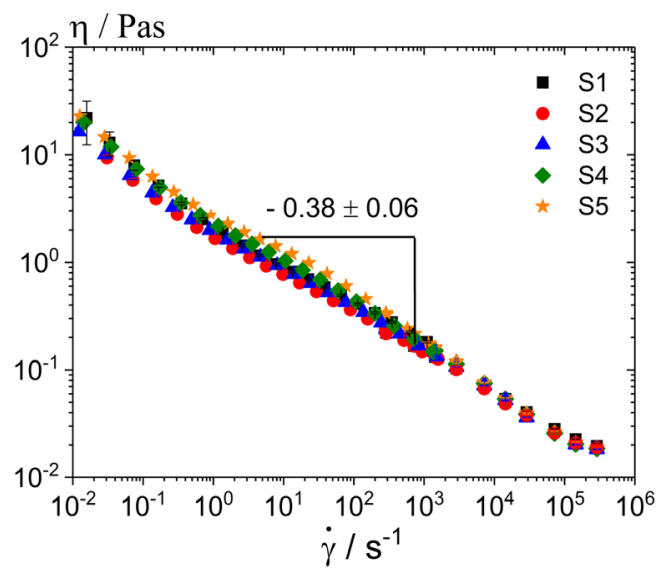

Fig. 2 Shear viscosity $\eta$ as a function of shear rate $\dot{\gamma}$ for Sterocoll mixtures $\mathrm{S} 1-\mathrm{S} 5$ as determined using rotational rheometry and capillary rheometry polymer concentration that is essentially constant for this series of samples. Gel content or physical crosslinking is relevant at low shear rates or frequencies. This is confirmed by the shear modulus data displayed in Fig. 3, where different viscoelastic properties can be observed in the low frequencies regime $\omega<10^{-1} \mathrm{rad} \mathrm{s}^{-1}$. Samples with a higher content of Sterocoll HT show larger characteristic relaxation time $\lambda_{\mathrm{s}}$, defined as the inverse of the angular frequency $\omega_{c}$ at which $\mathrm{G}^{\prime}=\mathrm{G}^{\prime \prime}$, indicating that the average apparent molecular weight increases with increasing Sterocoll HT fraction in the mixtures due to the increasing number of intermolecular hydrophobic associations. However, at the angular frequency $\omega>$ $10 \mathrm{rad} \mathrm{s}^{-1}, \mathrm{G}^{\prime}$ and $\mathrm{G}^{\prime \prime}$ converge and further increase according to the power-law $\mathrm{G}^{\prime} \approx \mathrm{G}^{\prime \prime} \sim \omega^{0.47}$. This exponent is clearly lower than the value expected from the Zimm model for polymers in a good solvent and may be a consequence of the hydrophobic associations.

Elongational flow properties of the Sterocoll mixtures were characterized using the CaBER method. The time evolution of the normalized filament diameter is shown in Fig. 4. While the shape of the curves is essentially the same for all samples, increasing the fraction of Sterocoll XT in the solution leads

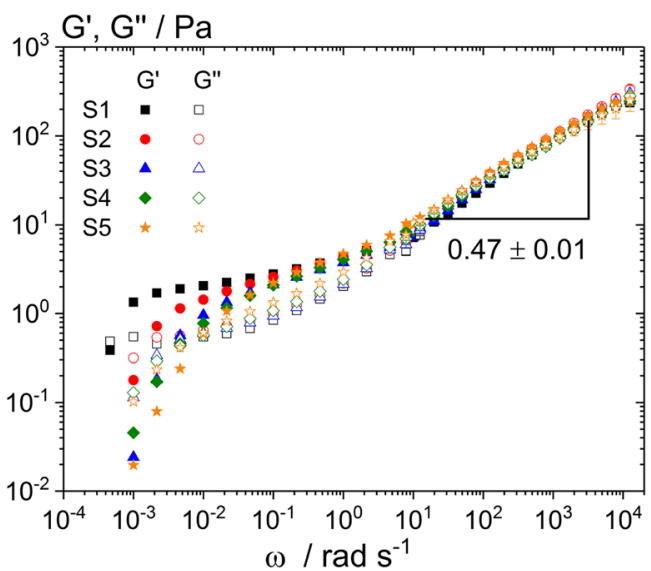

Fig. 3 Storage modulus $\mathrm{G}^{\prime}$ and loss modulus $\mathrm{G}^{\prime \prime}$ vs. angular frequency for Sterocoll mixtures 


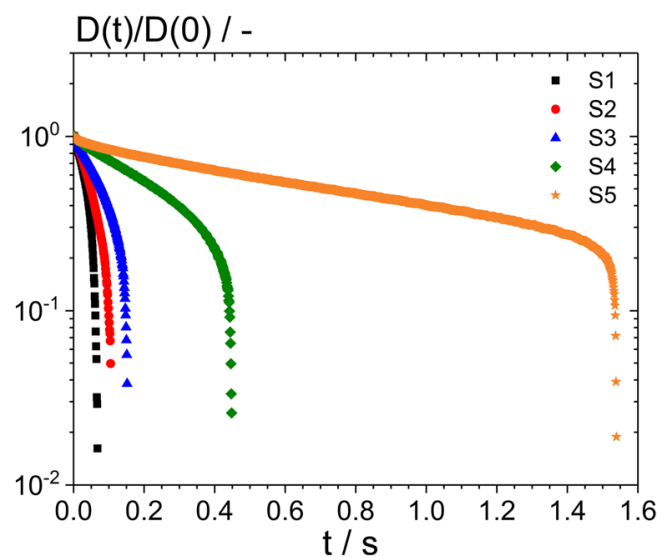

Fig. 4 Filament diameter $D(t)$ normalized by its initial value $D(0)$ immediately after the piston has reached its final position $h_{f}$ vs. filament thinning time for Sterocoll mixtures S1-S5

to an increase in the breakup time from $t_{\text {breakup }}=0.075 \mathrm{~s}$ for sample $\mathrm{S} 1$ to $t_{\text {breakup }}=1.55 \mathrm{~s}$ for sample S5. Figure 5 shows the corresponding strain rate plotted against the filament thinning time. All of the samples exhibit an exponential decay of $D(t)$, and correspondingly a constant strain rate varying between $\dot{\varepsilon}=2 \mathrm{~s}^{-1}$ for $\mathrm{S} 5$ and $\dot{\varepsilon}=50 \mathrm{~s}^{-1}$ for $\mathrm{S} 1$ over an extended period of time. Accordingly, in this elasto-capillary regime in which surface tension is balanced by elastic stresses, filament thinning is characterized by a single relaxation time $\lambda_{\mathrm{e}}$ that is calculated according to Eq. (2). This elasto-capillary thinning is followed by a terminal regime in which the filament diameter decays rapidly due to the finite extensibility of the polymer chains. We cannot determine a steady-state extensional viscosity for such weakly viscoelastic systems. Therefore, we characterize their elongational flow resistance in terms of $\lambda_{\mathrm{e}}$.

The shear and elongational relaxation time data for all of the Sterocoll mixtures are collected in Fig. 6. It is observed that an increase in the amount of Sterocoll XT leads to an increase in the elongational relaxation time $\lambda_{e}$ by almost two orders of magnitude. At the same time, $\lambda_{s}$, which is several

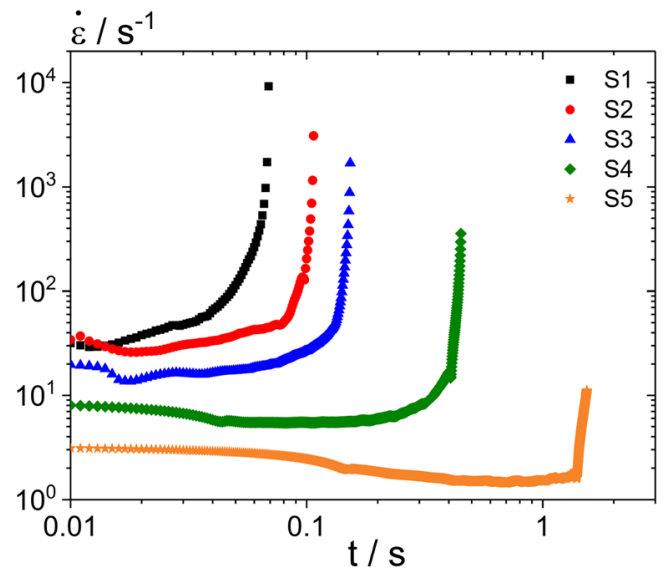

Fig. 5 Strain rate $\dot{\varepsilon}$ according to Eq. (1) vs. filament thinning time for Sterocoll mixtures S1-S5

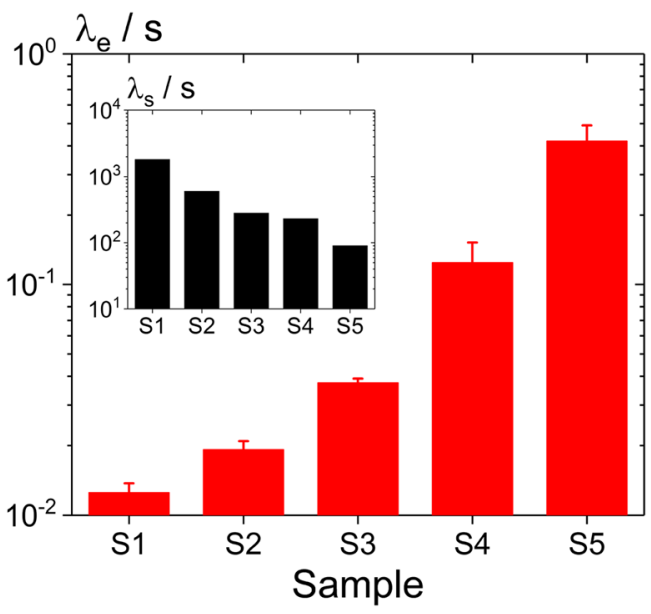

Fig. 6 Elongational relaxation time $\lambda_{e}$ of the Sterocoll mixtures S1-S5. Inset, shear relaxation time $\lambda_{s}$ for these Sterocoll mixtures

orders of magnitude higher than $\lambda_{e}$, decreases by approximately an order of magnitude, because the fraction of Sterocoll HT in the mixtures decreases. The low ratio $\lambda_{e} / \lambda_{s}$ is attributed to the intermolecular aggregates resulting from attractive interactions among the hydrophobic chain segments that cannot withstand the strong elongational flow. The strong increase in $\lambda_{e}$ with increasing fraction of Sterocoll XT is rationalized as follows: polymer molecules with strong intrachain hydrophobic attraction such as Sterocoll HT can hardly deform or transmit tensile stresses, and hence do not contribute much to the elongational flow resistance. This is different for linear chains of molecules with only few intrachain hydrophobic associations such as Sterocoll XT. These molecules strongly deform in elongational flow and consequently $\lambda_{e}$ increases with increasing fraction of Sterocoll XT in the solutions. Similar differences in the response to elongational deformation have been observed earlier by comparing the extensional viscosity of the solutions including various linear flexible polymers to the solutions of rigid molecules (Dexter 1996; Stelter et al. 2002) and particularly by Kheirandish et al. $(2008,2009)$ who investigated the shear and elongational relaxation times of similar ASE-type acrylic thickeners.

In summary, we have designed a series of aqueous solutions with different mixing ratios of two ASE-type acrylic thickeners differing in the fraction of copolymerized hydrophobic monomers, and hence the degree of inter- and intrachain hydrophobic association also known as physical crosslinks. These mixtures show pronounced shear thinning that is typical for many industrial coatings and exhibit essentially the same shear viscosity values particularly at high shear rates relevant for industrial coating operations. This model system is ideally suited to study the effect of elongational flow resistance on atomization, jetting, or dispensing of technical coating, ink, or adhesive formulations (Oswald et al. 2019). 


\section{Approach 2: adding a small amount of plate-like particles to highly shear-thinning thickener solutions}

A small amount of non-Brownian, strong anisotropic rod-like particles suspended in Newtonian fluids causes high tensile stresses during uniaxial extensional flows, whereas the resistance to shear flows is hardly affected (Weinberger and Goddard 1974). Here, we discuss how plate-like particles added to shear thinning, viscoelastic fluids alter the shear and elongational flow behavior. These oblate particles hardly affect shear viscosity as shown in an example illustrated in Fig. 7 for different suspensions of glass flakes with $A R \approx 25$ in thickener solution S3. This is expected based on the generalized Einstein relation for the relative viscosity $\eta_{r}$ of dilute suspensions including non-Brownian, hard anisotropic particles (Pabst et al. 2006):

$\frac{\eta_{\text {suspension }}}{\eta_{\text {solvent }}}=1+[\eta] \varphi$.

Inserting the intrinsic viscosity $[\eta]=3.13$ calculated by Hinch and Leal (1972) for oblate particles with high aspect ratio and for high rotary Peclet number (here $\mathrm{Pe}_{\mathrm{r}}>10^{10}$ ) leads to $\eta_{r}=1.11$ for $\varphi_{f}=3.5 \mathrm{vol} . \%$, which is in good agreement with our results at any given shear rate. A similar weak dependence of shear viscosity on $\varphi$ was already observed for aqueous suspension containing $5.0 \mathrm{vol} . \%$ of plate-like kaolin particles with $A R=12$ (Jogun and Zukoski 1996). Next, we discuss the elongational flow behavior of the glass flake suspension introduced above based on the CaBER experiments.

Generally, evaluation of CaBER data assumes a constant velocity within the filament cross-section and a velocity gradient in the axial direction. To check this assumption, we determined the true velocity field in a horizontally thinning filament of thickener solutions with different volume fractions

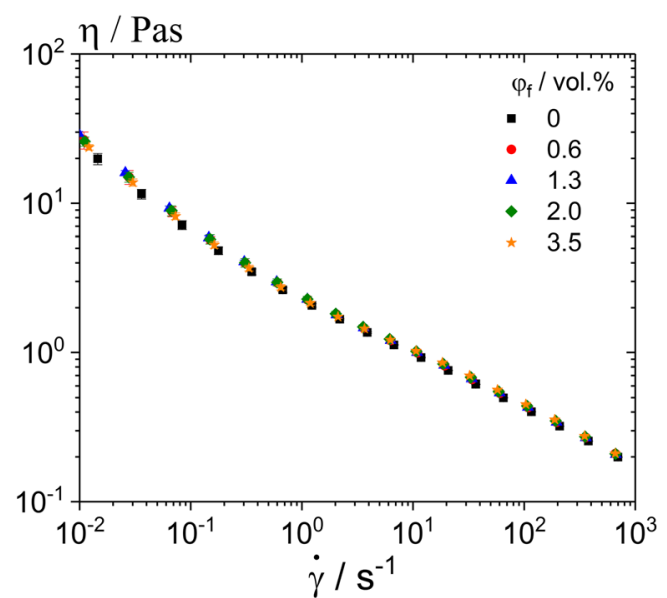

Fig. 7 Shear viscosity $\eta$ as a function of shear rate $\dot{\gamma}$ for a suspension of glass flakes in $\mathrm{S} 3$ differing in particle volume fraction $\varphi_{f}$ of glass flakes using PIV. For these measurements, a Sterocoll mixture S6 consisting of $1.0 \mathrm{wt} . \%$ Sterocoll HT and $1.0 \mathrm{wt} . \%$ Sterocoll XT was used and exhibited a breakup time of approximately $30 \mathrm{~s}$, which is sufficiently long to perform PIV. Figure 8 shows the axial velocity field for the thickener solution S6 including 0.6 vol.\% and 3.5 vol.\% glass flakes a few seconds after filament stretching was started. This corresponds to the elasto-capillary regime in which $\lambda_{\mathrm{e}}$ is determined. The filament diameter $\mathrm{D}(\mathrm{t})$ is still much larger than the geometrical dimensions of the suspended flakes. The focus could not be adjusted exactly to the stagnation point and thus, only positive axial velocity values are detected. Both filaments show almost constant axial velocity across the diameter. From this, we can conclude that the filament thinning follows a uniaxial extension, and no flow heterogeneities or instabilities occur during the capillary thinning of these suspensions as reported recently for dilute surfactant and biopolymer solutions (Recktenwald et al. 2019). The flakes apparently do not disturb the uniaxial extensional thinning as long as the filament is much larger than the flake dimension.

Figure 9 shows the time evolution of the filament diameter during the capillary thinning of S3-based suspensions with different amounts of glass flakes. An increase in the volume fraction of glass flakes results in a substantial increase in the filament breakup time. Again, a distinct elasto-capillarythinning regime occurred for all of the investigated suspensions and the slope of the normalized $D(t)$ curve decreases with increasing fraction of glass flakes, i.e., these particles slow down the thinning process. The strain rate drops from $\dot{\varepsilon}=22 \mathrm{~s}^{-1}$ for pure $\mathrm{S} 3$ to $\dot{\varepsilon}=5 \mathrm{~s}^{-1}$ for the suspension including 3.5 vol.\% glass flakes. Correspondingly, the characteristic elongational relaxation time $\lambda_{\mathrm{e}}$ increases from $31 \mathrm{~ms}$ to $131 \mathrm{~ms}$. In all cases, the elasto-capillary thinning is followed by a terminal regime characterized by rapid decay of the filament diameter $D(t)$. It should be noted that similar results, i.e., no effect of the suspended glass flakes on shear viscosity but a strong increase in the filament lifetime $t_{\text {breakup }}$ with $\varphi_{\mathrm{f}}$ were also found for the suspensions of the glass flakes in the Newtonian solvent glycerol. The corresponding data shown in Fig. 10 reveal an exponential scaling of $t_{\text {breakup }}$ with increasing $\varphi_{\mathrm{f}}$ irrespective of the chosen solvent. However, the increase is slightly weaker when the flakes are suspended in glycerol instead of the acrylic thickener solution S3. Utracki and Lara (1984) studied the effect of the mica flakes with $\mathrm{AR}=35$ suspended in a high-density polyethylene (HDPE) melt on extensional viscosity. Similar to the findings of the present study, an increase in the flake volume fraction up to 10 vol.\% resulted in an increase in the extensional viscosity by approximately one order of magnitude compared with the pure polymer. Theoretical work of Brenner (1974) demonstrated that the intrinsic extensional viscosity $\left[\eta_{e}\right]$ increases with increasing aspect ratio. Batchelor (1971) considered the 
Fig. 8 Velocity $v_{z}$ as a function of the axial position $z$ and radial position $r$ for a S6 with 0.6 vol.\% glass flakes after $3.8 \mathrm{~s}$ and $\mathbf{b} \mathrm{S} 6$ with 3.5 vol.\% glass flakes after $5 \mathrm{~s}$

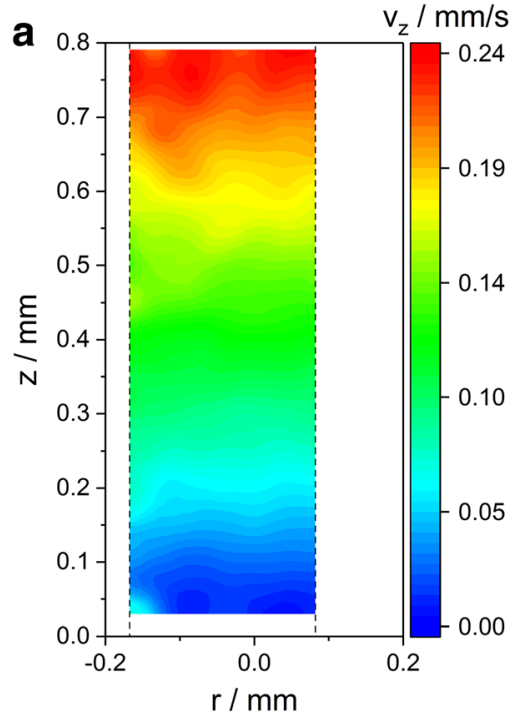

extensional viscosity in the semidilute regime for suspensions of rod-like particles in Newtonian fluids and found a more pronounced increase in the hydrodynamic stress in elongational flow than in shear flow. Weinberger and Goddard (1974) reported an order of magnitude increase in tensile stress when $1.3 \mathrm{vol} . \%$ of glass fibers with $\mathrm{AR}=1 / 57$ were added to a silicon oil and a polybutene solution, while shear viscosity increased by less than $10 \%$. To summarize, in accordance with theoretical considerations for anisotropic particles suspended in Newtonian fluids (Batchelor 1971; Hinch and Leal 1972; Brenner 1974) and experimental work on fiber suspensions in Newtonian fluids (Weinberger and Goddard 1974) and flakes suspended in polymer melts (Utracki and Lara 1984), our experimental data show that adding small fractions of plate-like particles to low viscosity, shearthinning fluids can be used to modify their elongational flow resistance at essentially constant shear viscosity.

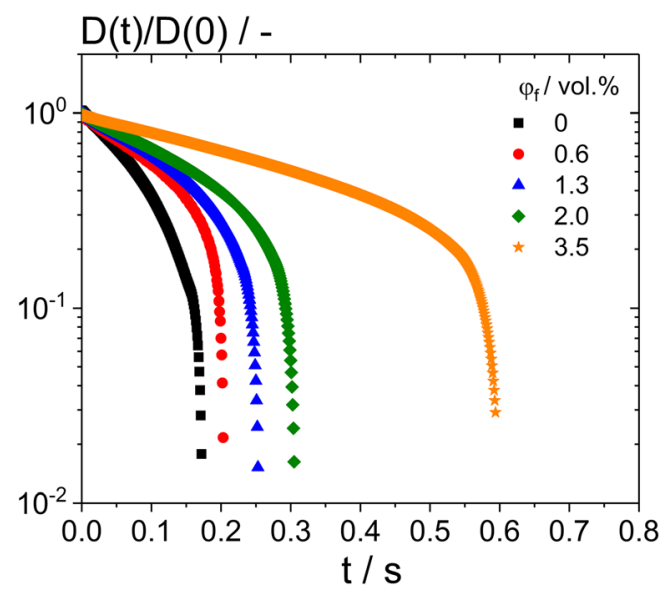

Fig. 9 Filament diameter $D(t)$ normalized by its initial value $D(0)$ immediately after the piston has reached its final position $h_{f}$ vs. filament thinning time for suspensions with different particle concentration $\varphi_{f}$
To provide evidence that this second approach of controlling elongational flow behavior independently from shear viscosity is also valid for complex, commercial fluids, experiments were performed employing waterborne automotive basecoats including different fractions of aluminum flakes. From Fig. 11, we observed that shear viscosity is hardly affected by the added aluminum flakes. Only the sample with 3.0 vol.\% particles exhibits a lower viscosity at shear rates $\dot{\gamma}$ $<1 \mathrm{~s}^{-1}$ and in the shear rate range between $10^{3} \dot{\gamma}<10^{5} \mathrm{~s}^{-1}$. This may be attributed to specific interactions of the aluminum particles with at least one of the components of this proprietary multicomponent coating mixture. Our results are largely in agreement with our expectation for dilute suspensions of anisotropic particles in Newtonian fluids. Based on Eq. (3), relative viscosity $\eta_{r}=1.09$ is estimated for the basecoat

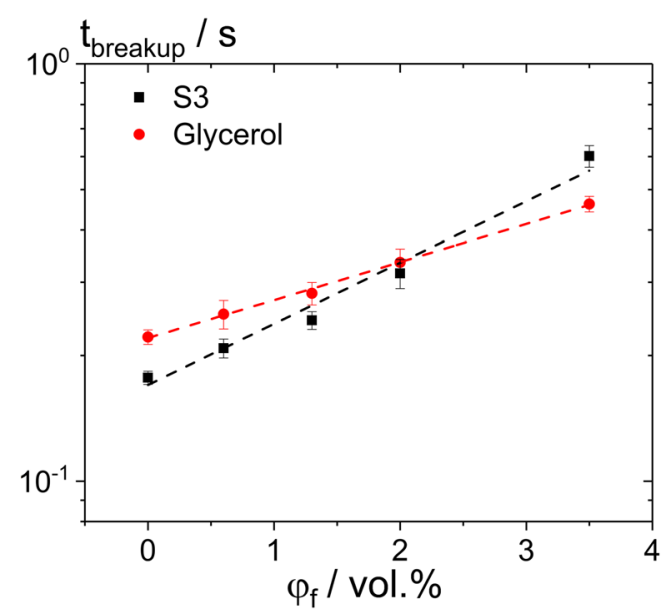

Fig. 10 Filament breakup time $t_{\text {breakup }}$ vs. volume fraction $\varphi_{f}$ of suspended glass flakes for acrylic thickener solution S3 (black squares) and glycerol (red circles). Dashed lines correspond to an exponential approach of the data 


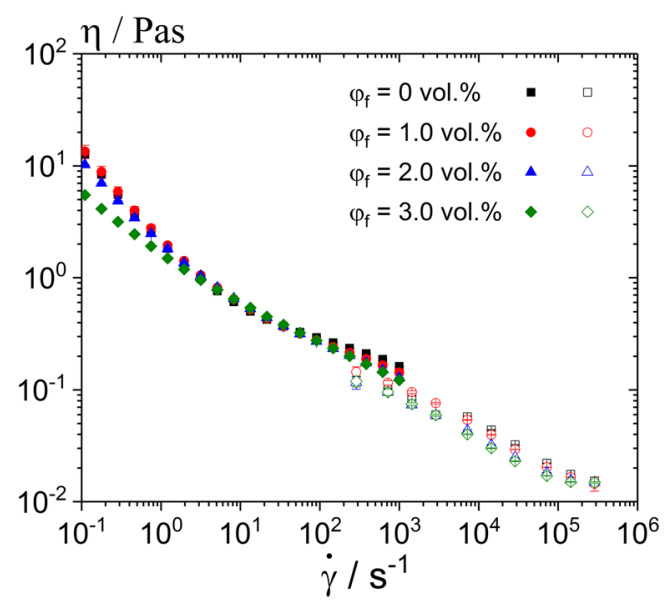

Fig. 11 Shear viscosity $\eta$ as a function of shear rate $\dot{\gamma}$ for waterborne automotive basecoats including different concentrations of aluminum flakes. Closed symbols refer to data from rotational rheometry, open symbols correspond to capillary rheometry data

including 3.0 vol. $\%$ aluminum flakes, again using $[\eta]=3.13$ for plate-like particles with $A R \gg 1$ at high rotary Peclet numbers.

The results of the CaBER experiments on the waterborne basecoats are plotted in Fig. 12. Again, an elasto-capillary thinning regime is observed followed by a terminal regime with a rapid diameter decay. A small increase in the particle volume fraction leads to pronounced deceleration of capillary thinning. Comparing the sample with 3.0 vol.\% aluminum flakes to the one without flakes, we observe a 2.5 -fold increase of the capillary breakup time corresponding to a drop of the strain rate from $\dot{\varepsilon}=26 \mathrm{~s}^{-1}$ to $\dot{\varepsilon}=10 \mathrm{~s}^{-1}$ and hence, the elongational relaxation time increases from $\lambda_{e}=26 \mathrm{~ms}$ to $\lambda_{e}=66 \mathrm{~ms}$.

In conclusion, this second approach of controlling the elongational flow behavior without affecting shear viscosity

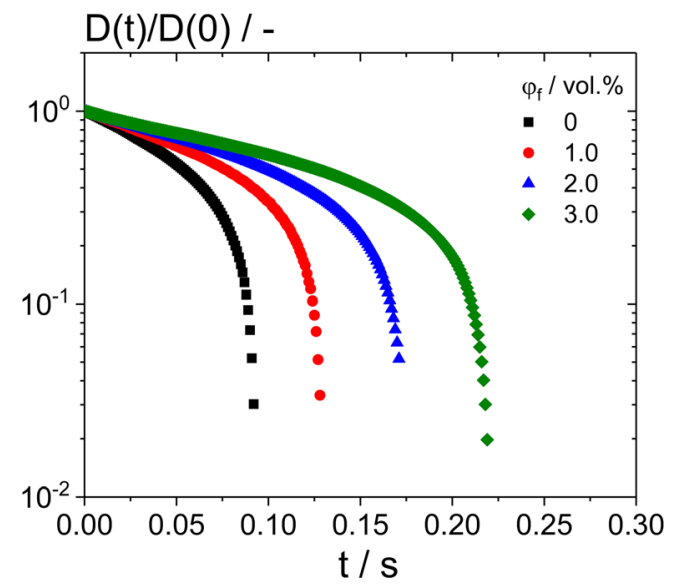

Fig. 12 Filament diameter $D(t)$ normalized by its initial value $D(0)$ immediately after the piston has reached its final position $h_{f}$ vs. filament thinning time for waterborne automotive basecoats with different concentration of aluminum flakes

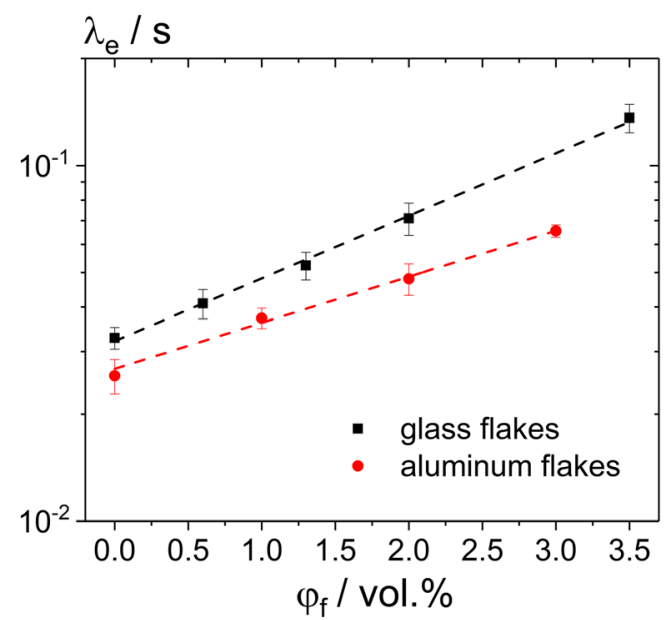

Fig. 13 Elongational relaxation time $\lambda_{e}$ vs. volume fraction $\varphi_{f}$ of suspended flakes for thickener solution S3 (black squares) and waterborne automotive basecoat (red circles). Dashed lines correspond to an exponential fit according to Eq. (4)

by introducing a small amount of plate-like particles is also valid for an industrially used waterborne basecoat. Figure 13 shows the elongational relaxation time plotted vs. the volume fraction of the flakes for the acrylic thickener solution and for the waterborne basecoat. For both materials, we find an exponential increase of $\lambda_{e}$ with the particle volume fraction $\varphi_{\mathrm{f}}$ according to:

$\lambda_{e}=A \cdot \exp \left(B \cdot \varphi_{f}\right)$

The corresponding fit parameters are summarized in Table 3. However, this exponential increase is less pronounced for the aluminum flakes than for the glass flakes despite the much lower aspect ratio of the latter. In light of Batchelor's (1971) calculations for rod-like particles, a stronger increase of the elongational flow resistance is expected when particles with higher aspect ratio are suspended. This is clearly not true here and we speculate that the effect of the aluminum flakes on the elongational flow behavior of the automotive basecoat is weakened by the complex interactions with different solvents, surfactants, and rheology modifiers included in this multicomponent fluid. We could not compare the effect of both flake types in a single solvent, because we were not able to create stable homogeneous suspensions of aluminum flakes in the acrylic thickener solutions or a stable suspension of glass flakes in the automotive basecoat.

\section{Summary and conclusion}

Control of the shear and elongational viscosities of complex fluids independently of each other in order to meet different 
Table 3 Fit parameters of Eq. (4)

for different suspensions

including plate-like particles

\begin{tabular}{lll}
\hline Type of particle & A & B \\
\hline Glass flakes in S3 & $0.032 \pm 0.001$ & $0.406 \pm 0.008$ \\
Aluminum flakes in waterborne basecoat & $0.027 \pm 0.001$ & $0.298 \pm 0.013$ \\
\hline
\end{tabular}

demands during manufacturing or application, e.g., of coatings or adhesives is still a profound technological challenge.

In this study, we present two approaches that allow for a large variation of the elongational relaxation time at an almost constant shear viscosity for technically relevant shear-thinning fluids. One way to achieve this objective is to mix a rigid polymer with a small amount of a more flexible polymer. We used two industrial ASE-type acrylic thickeners, namely, Sterocoll® HT and Sterocoll® XT. Due to a larger number of inter- and intramolecular hydrophobic associations, Sterocoll HT chains or aggregates are much more rigid than the Sterocoll XT molecules. Adding a small fraction of flexible Sterocoll XT to Sterocoll HT leads to a decrease in the longest shear relaxation time, whereas the steady shear viscosity is unaffected, particularly at the high shear rates relevant for many technical processes and coating operations. However, the flexible chains strongly deform in elongational flow and hence, the elongational relaxation time can be increased from $\lambda_{e}=12.5 \mathrm{~ms}$ for the pure solution including $2.3 \mathrm{wt} . \%$ Sterocoll HT up to $\lambda_{e}=420 \mathrm{~ms}$ for the mixture containing 1.6 wt.\% Sterocoll HT and 0.4 wt.\% Sterocoll XT.

The second approach is based on the phenomenon that suspensions of anisotropic particles in Newtonian fluids exhibit higher resistance to imposed elongational deformation than to shear deformation (Batchelor 1971; Shaqfeh and Fredrickson 1990). This encouraged us to add small amounts of plate-like particles into a shear-thinning acrylic thickener solution and commercial coating formulation. Increasing the volume fraction of glass flakes up to $3.5 \mathrm{vol} \%$ in the acrylic thickener solution S3 resulted in a 4-fold increase of the elongational relaxation time without significantly affecting the shear flow properties. Similar results were also found for industrial automotive waterborne basecoats including aluminum flakes, and in both cases, $\lambda_{e}$ increases exponentially with increasing flake concentration.

PIV revealed uniform uniaxial thinning across the filament diameter during the CaBER experiments on the suspensions of glass flakes in shear-thinning acrylic thickener solutions. This confirms that the delayed capillary thinning is not due to heterogeneous flow or even flow instabilities, but rather is due to the alignment of the plate-like particles.

Technological processes such as atomization, spraying, curtain and blade coating, or inkjet and screen printing, all include elongational deformation of the processed fluids. Rational improvement of these processes or the processed materials may benefit from our investigations regarding the control of the elongational flow resistance at a constant shear viscosity. The model system introduced here can be used for systematic studies of how the elongational flow resistance of the processed fluids affects these printing or coating operations. Further systematic investigations regarding the dependence of the elongational viscosity on the aspect ratio and volume fraction for the suspensions including oblate particles may be stimulated. To date, rheological research was focused on suspensions containing rods or fibers. Plate-like particle suspensions, however, are relevant not only for achieving metallic or pearlescent effects in coatings but also for processing clay minerals or composite materials with high thermal or electrical conductivity.

Acknowledgments The authors would like to thank Georg Wigger and Daniel Briesenick, both from the BASF Coatings GmbH for enabling this investigation and BASF SE and Eckart GmbH for providing the materials. Additionally, we would like to thank Steffen Recktenwald for his help in performing the PIV measurements.

\section{References}

Anna SL, McKinley GH (2001) Elasto-capillary thinning and breakup of model elastic liquids. J Rheol (N Y N Y) 45:115-138. https://doi. org/10.1122/1.1332389

Anna SL, McKinley GH, Nguyen DA et al (2001) An interlaboratory comparison of measurements from filament-stretching rheometers using common test fluids. J Rheol (N Y N Y) 45:83-114. https:// doi.org/10.1122/1.1332388

Arnolds O, Buggisch H, Sachsenheimer D, Willenbacher N (2010) Capillary breakup extensional rheometry (CaBER) on semi-dilute and concentrated polyethyleneoxide (PEO) solutions. Rheol Acta 49:1207-1217. https://doi.org/10.1007/s00397-010-0500-7

Bartolo D, Boudaoud A, Narcy G, Bonn D (2007) Dynamics of nonNewtonian droplets. Phys Rev Lett 99:174502. https://doi.org/10. 1103/PhysRevLett.99.174502

Batchelor GK (1971) The stress generated in a non-dilute suspension of elongated particles by pure straining motion. J Fluid Mech 46:813829. https://doi.org/10.1017/S0022112071000879

Bazilevskii AV, Entov VM, Lerner MM, Rozhkov AN (1997) Failure of polymer solution filaments. Polym Sci Ser A Chem Phys 39:316324

Bazilevsky AV, Entov VM, Rozhkov AN (2011) Breakup of a liquid bridge as a method of rheological testing of biological fluids. Fluid Dyn 46:613-622. https://doi.org/10.1134/S0015462811040119

Bergeron V, Bonn D, Martin JY, Vovelle L (2000) Controlling droplet deposition with polymer additives. Nature 405:772-775. https://doi. org/10.1038/35015525

Brenner H (1974) Rheology of a dilute suspension of axisymmetric Brownian particles. Int J Multiph Flow 1:195-341. https://doi.org/ 10.1016/0301-9322(74)90018-4

Christanti Y, Walker LM (2001) Surface tension driven jet break up of strain-hardening polymer solutions. J Nonnewton Fluid Mech 100: 9-26. https://doi.org/10.1016/S0377-0257(01)00135-5 
Christanti Y, Walker LM (2002) Effect of fluid relaxation time of dilute polymer solutions on jet breakup due to a forced disturbance. $\mathrm{J}$ Rheol (N Y N Y) 46:733-748. https://doi.org/10.1122/1.1463418

Clasen C (2010) Capillary breakup extensional rheometry of semi-dilute polymer solutions. Korea-Australia Rheol J 5:331-338

Clasen C, Plog JP, Kulicke W-M, Owens M, Macosko C, Scriven LE, Verani M, McKinley GH (2006) How dilute are dilute solutions in extensional flows? J Rheol (N Y N Y) 50:849-881. https://doi.org/ $10.1122 / 1.2357595$

Cogswell FN (1972) Measuring the extensional rheology of polymer melts. Trans Soc Rheol 16:383-403. https://doi.org/10.1122/1. 549257

Crassous JJ, Régisser R, Ballauff M, Willenbacher N (2005) Characterization of the viscoelastic behavior of complex fluids using the piezoelastic axial vibrator. J Rheol (N Y N Y) 49:851-863. https://doi.org/10.1122/1.1917843

Dexter RW (1996) Measurement of extensional viscosity of polymer solutions and its effects on atomization from a spray nozzle. At Sprays 6:167-191. https://doi.org/10.1615/AtomizSpr.v6.i2.30

Entov VM, Hinch EJ (1997) Effect of a spectrum of relaxation times on the capillary thinning of a filament of elastic liquid. J Nonnewton Fluid Mech 72:31-53. https://doi.org/10.1016/S0377-0257(97) 00022-0

Fernando RH, Xing LL, Glass JE (2000) Rheology parameters controlling spray atomization and roll misting behavior of waterborne coatings. Prog Org Coatings 40:35-38. https://doi.org/10.1016/S03009440(00)00112-0

Fuller GG, Cathey CA, Hubbard B, Zebrowski BE (1987) Extensional viscosity measurements for low-viscosity fluids. J Rheol (N Y N Y) 31:235-249. https://doi.org/10.1122/1.549923

Greiciunas E, Wong J, Gorbatenko I, Hall J, Wilson MCT, Kapur N, Harlen OG, Vadillo D, Threlfall-Holmes P (2017) Design and operation of a Rayleigh Ohnesorge jetting extensional rheometer (ROJER) to study extensional properties of low viscosity polymer solutions. J Rheol (N Y N Y) 61:467-476. https://doi.org/10.1122/1. 4979099

Gupta K, Chokshi P (2017) Stability analysis of bilayer polymer fiber spinning process. Chem Eng Sci 174:277-284. https://doi.org/10. 1016/j.ces.2017.08.029

Gupta RK, Nguyen DA, Sridhar T (2000) Extensional viscosity of dilute polystyrene solutions: effect of concentration and molecular weight. Phys Fluids 12:1296-1318. https://doi.org/10.1063/1.870383

Hinch EJ, Leal LG (1972) The effect of Brownian motion on the rheological properties of a suspension of non-spherical particles. J Fluid Mech 52:683-712. https://doi.org/10.1017/S002211207200271X

Hoath SD, Vadillo DC, Harlen OG, McIlroy C, Morrison NF, Hsiao WK, Tuladhar TR, Jung S, Martin GD, Hutchings IM (2014) Inkjet printing of weakly elastic polymer solutions. J Nonnewton Fluid Mech 205:1-10. https://doi.org/10.1016/j.jnnfm.2014.01.002

Hyun JC (1999) Draw resonance in polymer processing: a short chronology and a new approach. Korea-Aust Rheol J 11:279-285

Jimenez LN, Dinic J, Parsi N, Sharma V (2018) Extensional relaxation time, pinch-off dynamics, and printability of semidilute polyelectrolyte solutions. Macromolecules 51:5191-5208. https://doi.org/10. 1021/acs.macromol.8b00148

Jogun S, Zukoski CF (1996) Rheology of dense suspensions of plate-like particles. J Rheol (N Y N Y) 40:1211-1232. https://doi.org/10.1122/ 1.550798

Keshavarz B, Sharma V, Houze EC, Koerner MR, Moore JR, Cotts PM, Threlfall-Holmes P, McKinley GH (2015) Studying the effects of elongational properties on atomization of weakly viscoelastic solutions using Rayleigh Ohnesorge jetting extensional Rheometry (ROJER). J Nonnewton Fluid Mech 222:171-189. https://doi.org/ 10.1016/j.jnnfm.2014.11.004

Kheirandish S, Guybaidullin I, Wohlleben W, Willenbacher N (2008) Shear and elongational flow behavior of acrylic thickener solutions.
Rheol Acta 47:999-1013. https://doi.org/10.1007/s00397-0080292-1

Kheirandish S, Gubaydullin I, Willenbacher N (2009) Shear and elongational flow behavior of acrylic thickener solutions. Part II: effect of gel content. Rheol Acta 48:397-407. https://doi.org/10. 1007/s00397-008-0324-x

Kizior TE, Seyer FA (1974) Axial stress in elongational flow of fiber suspension. Trans Soc Rheol 18:271-285. https://doi.org/10.1122/ 1.549336

Kwag C, Vlachopoulos J (1991) An assessment of Cogswell's method for measurement of extensional viscosity. Polym Eng Sci 31:10151021. https://doi.org/10.1002/pen.760311404

Lampe J, DiLalla R, Grimaldi J, Rothstein JP (2005) Impact dynamics of drops on thin films of viscoelastic wormlike micelle solutions. J Nonnewton Fluid Mech 125:11-23. https://doi.org/10.1016/j. jnnfm.2004.08.012

Le Meins JF, Moldenaers P, Mewis J (2003) Suspensions of monodisperse spheres in polymer melts: particle size effects in extensional flow. Rheol Acta 42:184-190. https://doi.org/10.1007/s00397-0020270-y

Mansour A, Chigier N (1995) Air-blast atomization of non-Newtonian liquids. J Nonnewton Fluid Mech 58:161-194. https://doi.org/10. 1016/0377-0257(95)01356-Z

McKinley GH, Tripathi A (2000) How to extract the Newtonian viscosity from capillary breakup measurements in a filament rheometer. J Rheol (N Y N Y) 44:653-670. https://doi.org/10.1122/1.551105

Meissner J (1985) Rheometry of polymer melts. Annu Rev Fluid Mech 17:45-64. https://doi.org/10.1146/annurev.fluid.17.1.45

Mewis J, Metzner AB (1974) The rheological properties of suspensions of fibres in Newtonian fluids subjected to extensional deformations. J Fluid Mech 62:593. https://doi.org/10.1017/S0022112074000826

Mueller S, Llewellin EW, Mader HM (2010) The rheology of suspensions of solid particles. Proc R Soc A Math Phys Eng Sci 466:12011228. https://doi.org/10.1098/rspa.2009.0445

Ng SL, Mun RP, Boger DV, James DF (1996) Extensional viscosity measurements of dilute solutions of various polymers. J Nonnewton Fluid Mech 65:291-298. https://doi.org/10.1016/ 0377-0257(96)01463-2

Niedzwiedz K, Arnolds O, Willenbacher N, Brummer R (2009) How to characterize yield stress fluids with capillary breakup extensional rheometry (CaBER)? Appl Rheol 19:1-10. https://doi.org/10. 3933/ApplRheol-19-41969

Ochowiak M, Broniarz-Press L, Rozanska S, Rozanski J (2012) The effect of extensional viscosity on the effervescent atomization of polyacrylamide solutions. J Ind Eng Chem 18:2028-2035. https:// doi.org/10.1016/j.jiec.2012.05.023

Oswald W, Gödeke L, Ehrhard P, Willenbacher N (2019) An experimental study of the influence of elongational flow behavior and pigmentation on the atomization with a high-speed rotary bell atomizer manuscript in preparation

Pabst W, Gregorová E, Berthold C (2006) Particle shape and suspension rheology of short-fiber systems. J Eur Ceram Soc 26:149-160. https://doi.org/10.1016/j.jeurceramsoc.2004.10.016

Padmanabhan M, Macosko CW, Padmanabhan M (1997) Extensional viscosity from entrance pressure drop measurements. Rheol Acta 36:144-151. https://doi.org/10.1007/BF00366820

Papanastasiou TC, Macosko CW, Scriven LE, Chen Z (1987) Fiber spinning of viscoelastic liquid. AICHE J 33:834-842. https://doi.org/10. 1002/aic.690330516

Recktenwald SM, Haward SJ, Shen AQ, Willenbacher N (2019) Heterogeneous flow inside threads of low viscosity fluids leads to anomalous long filament lifetimes. Sci Rep 9:7110. https://doi.org/ 10.1038/s41598-019-43590-z

Rodd LE, Scott TP, Cooper-White JJ, McKinley GH (2005) Capillary break-up rheometry of low-viscosity elastic fluids. Appl Rheol 15: 12-27. https://doi.org/10.3933/ApplRheol-15-12 
Rothstein JP, McKinley GH (2002) A comparison of the stress and birefringence growth of dilute, semi-dilute and concentrated polymer solutions in uniaxial extensional flows. J Nonnewton Fluid Mech 108:275-290. https://doi.org/10.1016/S0377-0257(02)00134-9

Rubinstein M, Colby RH (2003) Polymer physics. Oxford University Press, Oxford

Sachsenheimer D, Hochstein B, Buggisch H, Willenbacher N (2012) Determination of axial forces during the capillary breakup of liquid filaments - the tilted CaBER method. Rheol Acta 51:909-923. https://doi.org/10.1007/s00397-012-0649-3

Sachsenheimer D, Hochstein B, Willenbacher N (2014) Experimental study on the capillary thinning of entangled polymer solutions. Rheol Acta 53:725-739. https://doi.org/10.1007/s00397-014-07898

Santamaría-Holek I, Mendoza CI (2010) The rheology of concentrated suspensions of arbitrarily-shaped particles. J Colloid Interface Sci 346:118-126. https://doi.org/10.1016/j.jcis.2010.02.033

Shaqfeh ESG, Fredrickson GH (1990) The hydrodynamic stress in a suspension of rods. Phys Fluids A Fluid Dyn 2:7-24. https://doi. org/10.1063/1.857683

Spearot JA, Metzner AB (1972) Isothermal spinning of molten polyethylenes. Trans Soc Rheol 16:495-518. https://doi.org/10.1122/1. 549262

Spiegelberg SH, Ables DC, McKinley GH (1996) The role of end-effects on measurements of extensional viscosity in filament stretching rheometers. J Nonnewton Fluid Mech 64:229-267. https://doi.org/10. 1016/0377-0257(96)01439-5

Stelter M, Brenn G, Yarin a L et al (2000) Validation and application of a novel elongational device for polymer solutions. J Rheol (N Y N Y) 44:595-616. https://doi.org/10.1122/1.551102

Stelter M, Brenn G, Yarin AL, Singh RP, Durst F (2002) Investigation of the elongational behavior of polymer solutions by means of an elongational rheometer. J Rheol (N Y N Y) 46:507-527. https:// doi.org/10.1122/1.1445185

Tembely M, Vadillo D, Mackley MR, Soucemarianadin A (2012) The matching of a "one-dimensional" numerical simulation and experiment results for low viscosity Newtonian and non-Newtonian fluids during fast filament stretching and subsequent break-up. J Rheol (N Y N Y) 56:159-183. https://doi.org/10.1122/1.3669647

Teraoka I (2002) Polymer solutions. John Wiley \& Sons, Inc., New York, USA
Thielicke W, Stamhuis EJ (2014) PIVlab - towards user-friendly, affordable and accurate digital particle image velocimetry in MATLAB. J Open Res Softw 2. https://doi.org/10.5334/jors.bl

Thompson JC, Rothstein JP (2007) The atomization of viscoelastic fluids in flat-fan and hollow-cone spray nozzles. J Nonnewton Fluid Mech 147:11-22. https://doi.org/10.1016/j.jnnfm.2007.06.004

Tirtaatmadja V, Sridhar T (1993) A filament stretching device for measurement of extensional viscosity. J Rheol (N Y N Y) 37:10811102. https://doi.org/10.1122/1.550372

Tirtaatmadja V, McKinley HG, Cooper-White JJ (2006) Drop formation and breakup of low viscosity elastic fluids: effects of molecular weight and concentration. Phys Fluids 18:043101. https://doi.org/ 10.1063/1.2190469

Tropea C, Yarin AL, Foss JF (2007) Springer handbook of experimental fluid mechanics, 1st edn. Springer-Verlag, Berlin Heidelberg

Trouton FT (1906) On the coefficient of viscous traction and its relation to that of viscosity. Proc R Soc A Math Phys Eng Sci 77:426-440. https://doi.org/10.1098/rspa.1906.0038

Utracki LA, Lara J (1984) Extensional flow of mica-filled polyethylene. Polym Compos 5:44-51. https://doi.org/10.1002/pc.750050109

Vadillo DC, Tuladhar TR, Mulji AC, Jung S, Hoath SD, Mackley MR (2010) Evaluation of the inkjet fluid's performance using the "Cambridge Trimaster" filament stretch and break-up device. J Rheol (N Y N Y) 54:261-282. https://doi.org/10.1122/1.3302451

Weinberger CB, Goddard JD (1974) Extensional flow behavior of polymer solutions and particle suspensions in a spinning motion. Int $\mathrm{J}$ Multiph Flow 1:465-486. https://doi.org/10.1016/0301-9322(74) 90015-9

Williams PA, English RJ, Blanchard RL, Rose SA, Lyons L, Whitehead M (2008) The influence of the extensional viscosity of very low concentrations of high molecular mass water-soluble polymers on atomisation and droplet impact. Pest Manag Sci 64:497-504. https:// doi.org/10.1002/ps. 1523

Xing LL, Glass JE, Fernando RH (1999) Parameters influencing the spray behavior of waterborne coatings. J Coatings Technol 71:37-50. https://doi.org/10.1007/BF02697898

Publisher's note Springer Nature remains neutral with regard to jurisdictional claims in published maps and institutional affiliations. 